\title{
The use of multiple sources of social information in contest behavior: testing the social cognitive abilities of a cichlid fish
}

\author{
Takashi Hotta ${ }^{1 *}$, Tomohiro Takeyama ${ }^{1,2}$, Dik Heg ${ }^{3}$, Satoshi Awata ${ }^{4}$, Lyndon A. Jordan ${ }^{1,5}$ \\ and Masanori Kohda ${ }^{1}$ \\ ${ }^{1}$ Animal Society, Department of Biology and Geosciences, Graduate School of Science, Osaka City University, Osaka, Japan, \\ ${ }^{2}$ Department of Biosphere-Geosphere Science, Faculty of Biosphere-Geosphere Science, Okayama University of Science, \\ Okayama, Japan, ${ }^{3}$ Clinical Trials Unit, Department of Clinical Research, University of Bern, Bern, Switzerland, ${ }^{4}$ Sado Marine \\ Biological Station, Faculty of Science, Niigata University, Niigata, Japan, ${ }^{5}$ Department of Collective Behaviour, Max Planck \\ Institute for Ornithology, Konstanz, Germany
}

OPEN ACCESS

Edited by:

Peter Schausberger, University of Natural Resources and Life Sciences, Vienna, Austria

Reviewed by: Giovanni Benelli,

University of Pisa, Italy Adam Reddon,

McGill University, Canada

*Correspondence: Takashi Hotta Department of Biology and Geosciences, Graduate School of

Science, Osaka City University,

Sugimoto 3-3-138, Sumiyoshi,

Osaka 558-8585, Japan

takasi712000@yahoo.co.jp

Specialty section:

This article was submitted to Behavioral and Evolutionary Ecology,

a section of the journa

Frontiers in Ecology and Evolution

Received: 02 April 2015

Accepted: 07 July 2015

Published: 03 August 2015

Citation:

Hotta T, Takeyama T, Heg D, Awata S, Jordan LA and Kohda M (2015) The

use of multiple sources of social

information in contest behavior: testing the social cognitive abilities of a cichlid fish. Front. Ecol. Evol. 3:85 doi: 10.3389/fevo.2015.00085
Theory suggests that living in large social groups with dynamic social interactions often favors the evolution of enhanced cognitive abilities. Studies of how animals assess their own contest ability commonly focus on a single cognitive task, and little is known about the diversity or co-occurrence of cognitive abilities in social species. We examined how a highly social cichlid fish Julidochromis transcriptus uses four major cognitive abilities in contest situations; direct experience, winner/loser effects, social eavesdropping and transitive inference (TI). We conducted experiments in which fish assessed the social status of rivals after either direct physical contests or observed contests. Individuals used direct information from a previous physical encounter to re-establish dominance without additional contact, but winner/loser effects were not observed. Social eavesdropping alone was ruled out, but we found that transitive reasoning was used to infer social dominance of other individuals of unknown status. Our results suggest that in stable hierarchical social groups, estimations of contest ability, based on individual recognition pathways such as $\mathrm{Tl}$ and direct experience, are more prevalent than social eavesdropping or winner/loser effects. We suggest that advanced cognitive abilities might be widespread among highly social fishes, but have previously gone undetected.

Keywords: Julidochromis transcriptus, individual recognition, winner and loser effect, transitive inference, eavesdropping, social cognitive ability

\section{Introduction}

Throughout the animal kingdom, contests over limited resources (e.g., mate, territory, and food) are widespread but may entail high costs in terms of time, energy and risk of injury (Huntingfold and Turner, 1987). Behavior in iterated animal contests may be influenced by prior experiences (Hsu et al., 2006), potentially reducing costs or incidence of fighting in repeated encounters. Winning experiences, for example, tend to increase the probability of winning, e.g., winner effects, while losing experiences tend to decrease it, e.g., loser effects (Hsu et al., 2006; Benelli et al., 2015a,b). Furthermore, social eavesdropping, the ability to monitor the contests between unfamiliar individuals and use the information in subsequent 
aggressive interactions, may reduce fighting costs with unknown individuals (Oliveira et al., 1998).

In large social groups, especially those with dominance hierarchies (Jordan et al., 2010a,b) individuals frequently interact with familiar and unfamiliar group members, and also with unknown individuals from outside the group (e.g., Byrne and Whiten, 1988; Awata and Kohda, 2004; White and Gowan, 2013). Increased cognitive abilities will be favored if they allow individuals to indirectly infer their dominance relationship with unknown individuals and avoid costly aggressive interactions for dominance. An individual's place in the social order can be learned through direct interactions with others, including engaging with strangers. However, the costs of these interactions increase cumulatively with the size of the society since the likelihood of encountering stranger increases. By observing interactions between the stranger and known individuals, with whom a social relationship has already been established, an animal may predict their own relationship to unknown individuals. This component of cognitive ability is called transitive inference (TI;Hogue et al., 1996; Paz-y-Miño et al., 2004; Engh et al., 2005; Grosenick et al., 2007; MacLean et al., 2008; Vasconcelos, 2008 for review). A variety of studies have suggested that transitive inference may be used by higher vertebrates (e.g., Hogue et al., 1996; Peake et al., 2002; Peake and McGregor, 2004; Engh et al., 2005), and TI studies are mainly documented in animals with high sociality, such as apes, monkeys, hyena, chickens and corvids (Gillan, 1981; Bond et al., 2003; Paz-y-Miño et al., 2004; Engh et al., 2005), as well as a territorial and social fish (Grosenick et al., 2007; White and Gowan, 2013).

This raises the question whether transitive inference only occurs in those species with high cognitive abilities in general. While transitive inference is typically observed in animals with highly organized societies, the pattern of co-occurrence of other cognitive abilities such as social eavesdropping and winner/loser effects is not well understood (Hsu et al., 2006, 2011). Despite this, analyses of the contributions of different components of cognition are rare, with studies most often focussing on a single component of cognitive ability (Hsu et al., 2011). In some cases, multiple cognitive factors have been examined in a single species, with conflicting results. Despite using TI with direct experience and information gathered by eavesdropping, hens in stable groups do not use social eavesdropping alone (Hogue et al., 1996). Further, in Melanochromis auratus, a group living cichlid with strict linear dominance and high sociality, winner/loser effects do not operate (Chase et al., 2003). In contrast, in Siamese fighting fish, both social eavesdropping (e.g., Oliveira et al., 1998; McGregor et al., 2001) and winner/loser effects (e.g., Wallen and Wojciechowski-Metzlar, 1985) are observed, but there is no evidence that this species use transitive inference to infer social dominance.

While the general cognitive abilities of fish are not fully understood, there are reports of cognitive capacity in some fish that mirror those in higher vertebrates, e.g., social eavesdropping, individual recognition and winner/loser effects (Hsu et al., 2006, 2011; Alfieri and Dugatkin, 2011; Brown and Laland, 2011). The social intelligence hypothesis predicts that highly increased cognitive ability, e.g., recognizing group members and transitive inference will be favored in highly organized, large societies (Byrne and Whiten, 1988; Bshary et al., 2002; Bond et al., 2003; Brown et al., 2011; Bshary, 2011). Cooperatively breeding cichlids in Lake Tanganyika often have large groups associated with frequent social interactions with known and unknown individuals (e.g., Awata et al., 2005; Heg and Bachar, 2006; Wong and Balshine, 2010), which may favor the development of high cognitive abilities (e.g., Byrne and Whiten, 1988; Bond et al., 2003; Alfieri and Dugatkin, 2011). Julidochromis transcriptus and its congeners are cooperatively breeding cichlids with a highly organized social system (Awata and Kohda, 2004; Awata et al., 2005, 2006, 2008, 2010; Heg and Bachar, 2006; Kohda et al., 2009). Breeding groups consist of multiple unrelated males and females that frequently share paternity and cooperatively raise their brood (Awata et al., 2005). Breeding members frequently interact with each other, but strangers of varying social status often approach the territory or nests of the members (Awata and Kohda, 2004). This fish is therefore an ideal species in which to examine social cognitive capacity, and is amenable to experimental manipulation of social experiences. Here we use male $J$. transcriptus to examine and disentangle the effects of transitive inference, social eavesdropping, winner/loser effects and direct fighting experience to determine the relationship between these cognitive abilities and how they interact to influence social interactions.

\section{Methods}

\section{Study Animal and Housing Condition}

We obtained the subject fish J. transcriptus from commercial breeders. Experiments were conducted in our laboratory at Osaka City University. We used males (60-80 $\mathrm{mm}$ in total length) that had been kept with females in either $30 \times 40 \times 60 \mathrm{~cm}$ tanks of 20 individuals or $45 \times 40 \times 180 \mathrm{~cm}$ stock tanks with 60 individuals, both at $26^{\circ} \mathrm{C}$ under $12: 12 \mathrm{~h}$ light-dark cycles (Awata et al., 2006). Stock tanks contained multiple shelters of halfcut flower pots, stones and tiles put on coral gravel bottom. Water was aerated and filtered using sponge and external canister filters, and dissolved nitrogenous waste was never measured to be above acceptable levels. Commercial flake food (Tetramin) was provided twice a day. Prior to experiments, fish were successfully housed for more than 1 year, and frequently bred in captivity. Similar-sized males that had not encountered each other during this period were used in all experimental trials (average size

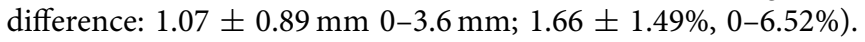
Glass aquariums $\left(30 \times 18 \times 20 \mathrm{~cm}^{3}\right)$ with gravel substrate were used for all experiments (Figure 1). Behavior was recorded with video cameras (HDR-CX370, Sony) in all experiment trials.

\section{Experiment Procedure}

Many previous studies of TI used 3-term series tasks using individuals A, B, and C (e.g., Bryant and Trabasso, 1971; Hogue et al., 1996; Peake and McGregor, 2004). In this study we also designed the 3-term series task using three individuals in test of TI, which are associated with several control experiments (Figure 2). We conducted five independent experiments to 
determine the effects of direct interactions and observation of interactions on subsequent aggressive and submissive behavior. Experiments consisted of a pre-phase I, an optional pre-phase II (depending on the treatment group), and a test phase (Figure 2). In preliminary experiments on direct physical contests, we observed that two size-matched fish put in a tank often continued to fight for at least $5 \mathrm{~min}$ after introduction, probably because of their similar body size and similar fighting ability. In these preliminary trials, dominance relationships took up to $15 \mathrm{~min}$ to become stable. Thus, we allowed $30 \mathrm{~min}$ to establish dominance after introducing two size-matched fish to arena tanks. We refer to individuals with established pairwise dominance as " $A$ " and "B," and an individual that had not yet established pairwise dominance with individual $\mathrm{A}$ as a stranger " $\mathrm{C}$ " and will use this terminology throughout the paper. During the test phase we recorded the following responses of each individual: (i) rushing time: time spent rapidly moving toward the glass barrier with open mouth in an aggressive fashion, and (ii) the time spent in each of three zones in test tank: the near, middle and far sections (each $6 \mathrm{~cm}$ width) from the border glass. Details of the experimental set-up and experimental sequences with predictions of each test are shown in Figures 1, 2. Sample sizes in respective experiments were 10 or 12 focal individuals.

In Experiment 1, we assessed how dominance during direct interactions in an "arena tank" affected subsequent behavior in a "test tank" (Figure 2A). In pre-phase I, two fish were placed in the arena tank for $30 \mathrm{~min}$ and their aggressive and submissive interactions were videotaped and monitored (10 pairs). The fish that performed aggressive acts toward and chased the other fish in a unidirectional manner or much more frequently than opponent during the last $15 \mathrm{~min}$ was regarded as winner and labeled "A." The losing fish was labeled "B." During the last half time, typically losers retreated from the winner if approached, and often showed submissive behavior or could flee from the winner. The winner did not continue to attack the loser when the latter performed subordinate displays, and therefore the losers were not persistently attacked or chased.

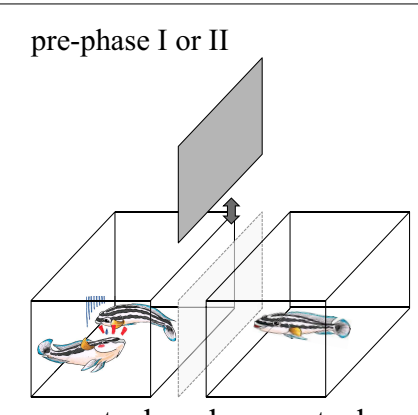

arena tank observer tank

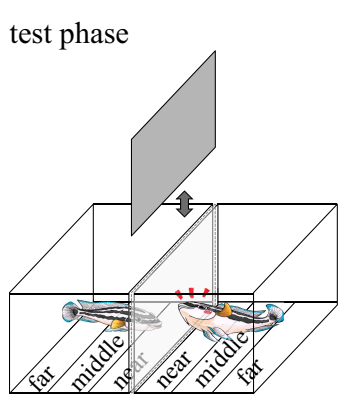

test tank

FIGURE 1 | Pictures of an arena tank, an observer tank (used in the pre-phase I and II) and test tanks (used in the test phase). These tanks all measured $30 \times 18 \times 20 \mathrm{~cm}^{3}(\mathrm{~W} \times \mathrm{D} \times \mathrm{H})$. The arena tank and the observer tank were separated with $7 \mathrm{~cm}$ distance. Movable opaque sheets are between the arena tank and the observer tank, and between the two adjacent test-tanks. The bottom of the test tanks was divided into three zones: near, middle and far zones, each having a width of $6 \mathrm{~cm}$

Fish $\mathrm{A}$ and $\mathrm{B}$ were then moved to each of the test tanks using a hand net, and visually isolated from each other using an opaque divider (Figures 1, 2A). Ten minutes after introduction, the opaque sheet was removed allowing fish to visually interact, and then their behaviors were recorded for $10 \mathrm{~min}$ (test phase). We analyzed total time (seconds) that fish attacked the glass divider with their mouth open and time in each of the three zones (near, middle, and far; Figure 1), using video recordings

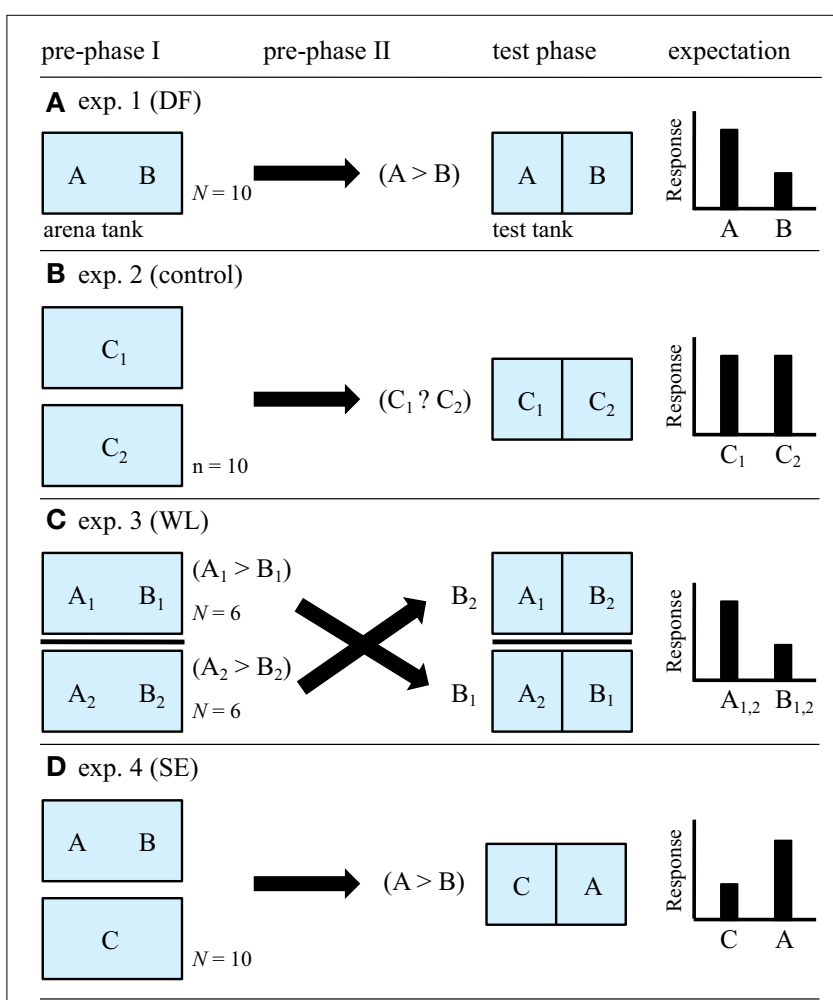

E exp. $5(\mathrm{TI})$

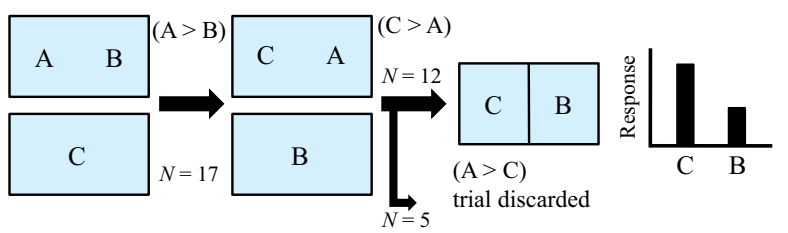

FIGURE 2 | Design of the experiments. All five experiments contained pre-phase I (and II if needed) and test phase. The expected responses (rushing time or time spent near the opponent, see text) are shown at the right when the predictions from the different hypotheses tested are supported. (A) Experiment $1 \mathrm{~A}$ and $\mathrm{B}$ had a direct encounter during the pre-phase and re-establish dominance during the test phase. (B) Experiment $2 \mathrm{C}_{1}$ and $\mathrm{C}_{2}$ had only visual contact in the pre-phase and establish dominance during the test phase. (C) Experiment 3 (testing winner/loser effects). A1 and A2 dominated B1 and B2 in arena tank, respectively. If $A_{1}$ and $A_{2}$ dominate against $B_{2}$ and $B_{1}$ against glass barrier in test phase, respectively, the loser-winner effect will operate. (D) Experiment 4 (testing eavesdropping hypothesis). Fish $\mathrm{C}$ observed interactions $\mathrm{A}>\mathrm{B}$. If the fish $\mathrm{C}$ exhibited submissive behaviors against $\mathrm{A}$, but if it exhibited aggressive behaviors against $B$, the prediction from eavesdropping hypothesis will be supported. (E) Experiment 5 (testing transitive inference hypothesis). Fish $\mathrm{C}$ observed interactions $\mathrm{A}>\mathrm{B}$, thereafter $\mathrm{B}$ observed interactions $\mathrm{C}>\mathrm{A}$. According to the transitive-inference hypothesis, fish $\mathrm{C}$ is expected to dominate over fish $\mathrm{B}$ against glass barrier. 
of the first $30 \mathrm{~s}$ on interaction. By analysing only the first $30 \mathrm{~s}$, we excluded possibility that one's behavior influences another's, i.e., collusion effects (Paz-y-Miño et al., 2004) . We expected the winner A to attack the glass barrier more and to spend more time in the near zone than the loser B during the test phase.

Experiment 2 acted as a control for Experiment 1. In pre-phase $\mathrm{I}$, two fish " $\mathrm{C}_{1}$ " and " $\mathrm{C}_{2}$ " were put in two tanks separated with a $7 \mathrm{~cm}$ distance for $30 \mathrm{~min}$ (Figure 2B), and they observed other fish but did not attack the glass barriers (10 pairs). These fish therefore had visual exposure, but did not have any experiences of direct contacts each other. These fish were then placed in the test tank and their behaviors were recorded as for the Experiment 1 (Figure 2B). We expected both fish to spend equal time in the near area during the test phase, because they are strangers to each other.

In Experiment 3, we tested the hypothesis that winning or losing a fight increases the probability of winning or losing again (winner/loser effects, Whitehouse, 1997; Oliveira et al., 2009). In pre-phase I, two arena tanks, each of which contained two fish, were used (Figure 2C). As in Experiment 1, we labeled the winners fish $A_{1}$ and $A_{2}$ and the losers fish $B_{1}$ and $B_{2}$ after 30min observations. In the test phase, $A_{1}$ and $B_{2}$ or $A_{2}$ and $B_{1}$ were allowed to be visually interacted and their behaviors were recorded (12 pairs). If winner/loser effects were operating, we would expect the previous winners $A_{1}$ and $A_{2}$ to exhibit more aggressive behaviors than the previous losers $B_{1}$ and $B_{2}$. In this experiment we used fish of size match, not the approach of random-selection (Hsu et al., 2006; Benelli et al., 2015a), because this Experiment 3 is also control for Experiment 5 where focal fish face the same size fish.

Experiment 4 was conducted to test whether fish that were allowed to observe fighting contests of other fish altered their behavior when they engaged those fish in subsequent fighting contests (eavesdropping hypothesis; Oliveira et al., 1998; Hsu et al., 2006, 2011). An observer fish C (in a separate observer tank) was allowed to observe interactions between two fish in an arena tank for $30 \mathrm{~min}$ (pre-phase I in Figure 2D). We then transferred the observer fish $\mathrm{C}$ into one compartment of a test tank and the winner fish $\mathrm{A}$ into another compartment (10 pairs). Their behaviors were recorded for $10 \mathrm{~min}$ (test phase in Figure 2D). If these animals use social eavesdropping to infer social dominance, the observer fish $\mathrm{C}$ would respond less aggressively when interacting with the winner A (Figure 2D).

In Experiment 5, we tested the hypothesis that J. transcriptus can use bi-directional transitive inference for inferring social dominance of strangers, using three individuals (12 triads). In the pre-phase I, two fish were placed in an arena tank, and watched by an observer fish $\mathrm{C}$ who was physically separated from the arena tank (Figure 2E). The observer fish $\mathrm{C}$ was allowed to watch the interactions between the two fish for $30 \mathrm{~min}$. The winner of the dyadic interaction was labeled $\mathrm{A}$, and the loser $\mathrm{B}$. The physically interacting fish $\mathrm{A}$ and $\mathrm{B}$ in arena tank ignored the fish $\mathrm{C}$ that observed the formers. In the pre-phase II, the winner $\mathrm{A}$ and the previous observer $\mathrm{C}$ was transferred into a new arena tank, while the loser $\mathrm{B}$ was moved to a new observer tank to be allowed to observe interactions between $\mathrm{A}$ and $\mathrm{C}$ from the separated tank (Figure 2E). As in the pre-phase I, we observed the two fish in the arena tank for $30 \mathrm{~min}$ and determined dominance relationships. If fish $\mathrm{C}$ was dominant over fish $\mathrm{A}$, the winner fish $\mathrm{C}$ and observer fish B were transferred to a test tank, and their behavior was recorded for $10 \mathrm{~min}$ (Figure 2E). If fish $\mathrm{C}$ was subordinate to fish $\mathrm{A}$ in the arena tank, we discarded the trial from the analyses. If fish $\mathrm{C}$ and $\mathrm{B}$ can use bidirectional transitive inference, it is predicted that both should correctly infer that $\mathrm{C}>\mathrm{B}$, despite that both fish had never experienced direct interactions between them. In this case, C and B would behave in an aggressive and submissive way during the test phase, respectively, which would be identical to the behavioral patterns of the winner $\mathrm{A}$ and the loser B in the test phase of Experiment 1.

\section{Ethical Notes}

This research adheres to the ASAB/ABS guidelines for the Use of Animals in Research (ASBS/ABS, 2014). All experiments were conducted in compliance with the Regulations on Animal Experiments in Osaka City University and the Japan Ethological Society. No permits were needed from Japanese government for experiments involving $J$. transcriptus.

We opted to handle fish without anesthetizing them because the effectiveness of anesthetizing in eliminating/reducing the stress of handling is not clear (Congleton, 2006). When we measured fish, we netted and placed the fish on top of several sheets of tissue saturated with water and covered the fish with another layer of wet tissue.

In escalated direct physical contests in arena tank, fish engaged in bouts of mouth biting where the two fish grasped each other's jaws and pushed each other (Sopinka et al., 2009). All contests for $30 \mathrm{~min}$ were videotaped and monitored by an observer. The observers had a rule to intervene and terminate contests if either of the fish appeared to suffer visible physical injury (e.g., scale loss, wounds, bleeding, or abnormal swimming behavior). However, no interventions were required because escalations in fight involving mouth biting were not observed to cause physical injuries to the fish. After contests were resolved, losers were often able to avoid attacks from the winners by sticking on the side or the corner of the aquarium although no refuge was put in the tank. In preliminary experiments, we observed that in the test tank containing a refuge (half of a small flower pot), some individuals stayed in refuges considerable time, which largely affected their reaction times and positions, and thus we did not put a refuge in test tanks. No refuge was put in arena tanks in order to make the condition of arena tank the same as test tanks.

None of the fish in arena tanks appeared to suffer physical damage from the contests. These losers were highly aggressive to unknown fish in subsequent test-phase experiments $10 \mathrm{~min}$ later (e.g., Experiments 2 and 3), showing that they were not damaged. After test phase experiments, fish were introduced to new stock containers, in which they took foods well and were all in good condition for more than 1 month, and were used in other experiments (Hotta et al., 2014).

\section{Statistical Methods}

Statistical analyses were performed using R. 2.13.2. Wilcoxon signed-rank tests and Mann-Whitney $U$-tests were used to compare rushing time within matched pairs and between fish 
in different experiments, respectively, during the test phase. Beta binominal generalized linear models (GLZ) were used for analyses of the proportion of time in the three zones during the test phase. Likelihood ratio tests were applied to test the significant effects of explanatory variables (fish group, zone, and its interaction).

\section{Results}

\section{Effect of Direct Experience}

In Experiment 1, the winner A more frequently attacked the loser $\mathrm{B}$ through the glass barrier (Wilcoxon signed-ranks test, $T=0.0$, $P=0.004$ ) and spent more time in the near zone than the loser $B$ (beta binomial GLZ, fish group $\times$ zone: $\chi_{2}^{2}=12.95, P=0.002$, Figure 3A). In contrast, in Experiment 2, rushing time and time in zones were not different between fish $C_{1}$ and $C_{2}$, which had not had direct contact experiences $(T=20.0, P=0.82$ in rushing time, fish group $\times$ zone: $\chi_{2}^{2}=0.24, P=0.89$; fish group: $\chi_{1}^{2}=$ $0.004, P=0.95$ in time in zones, Figure 3B). Comparing the two experiments, responses of the winner $\mathrm{A}$ in Experiment 1 and the strangers in Experiment 2 were not different (Mann-Whitney $U$ test, $z=-0.29 P=0.77$ in rushing time, fish group $\mathrm{x}$ zone: $\chi_{2}^{2}=5.31, P=0.07$, fish group: $\chi_{2}^{2}=0.04, P=0.85$ in time in zone, $N=20$, data of fish $C_{1}$ and fish $C_{2}$ in Experiment 2 were pooled). However, responses of the loser fish $\mathrm{B}$ in Experiment 1 and the strangers in Experiment 2 were significantly different $(z=-4.24, P<0.0001$ in rushing time, fish group $\mathrm{x}$ zone: $\chi_{2}^{2}=5.96, P=0.05$ in time in zone, $N=20$ ).

\section{Winner/Loser Effects}

In Experiment 3, we tested whether fish used winner/loser experience to infer dominance relationship. Contrary to the winner/loser effects expectation, neither rushing time nor the time in the three zones of the winners $\left(\mathrm{A}_{1}\right.$ and $\left.\mathrm{A}_{2}\right)$ differed from those of the losers $\left(\mathrm{B}_{1}\right.$ and $\mathrm{B}_{2}$; Wilcoxon signed-ranks test, $T=$ 22.0, $P=0.57$ in rushing time; beta binomial GLZ, fish group $\times$ zone: $\chi_{2}^{2}=1.58, P=0.45$, fish group: $\chi_{1}^{2}=0.07, P=0.79$ in time in zones, Figure $3 \mathrm{C}$ ).

\section{Social Eavesdropping}

In Experiment 4, we tested whether fish used social eavesdropping to infer dominance. Contrary to the social eavesdropping expectation, both rushing time and the time in the three zones of the fish $\mathrm{C}$, which had previously observed a contest between fish $\mathrm{A}$ and fish $\mathrm{B}$, did not differ from those of the opponent fish A, who defeated B (Wilcoxon signed-ranks test, $T$ $=24.0, P=0.77$ in rushing time; beta binomial GLZ, fish group $\times$ zone: $\chi_{2}^{2}=5.83, P>0.05$, fish group: $\chi^{2}=0.50, P=0.48$ in time in zones, Figure 3D) Comparing across experiments, rushing time and duration in each zone were not different between observer $\mathrm{C}$ of this experiment and observer $\mathrm{C}_{1}$ and $\mathrm{C}_{2}$ in Experiment 2 (Mann-Whitney $U$-test, $z=0.59 P=0.69$ in rushing time, fish group $\times$ zone: $\chi_{2}^{2}=0.75, P=0.69$, fish group: $\chi_{1}^{2}=0.05, P=0.82$ in time in zone, $N=20$ for both experiments, data of fish $\mathrm{C}$ against $\mathrm{A}$ in Experiment 4 and fish $\mathrm{C}_{1}$ and fish $\mathrm{C}_{2}$ in Experiment 2 were combined).

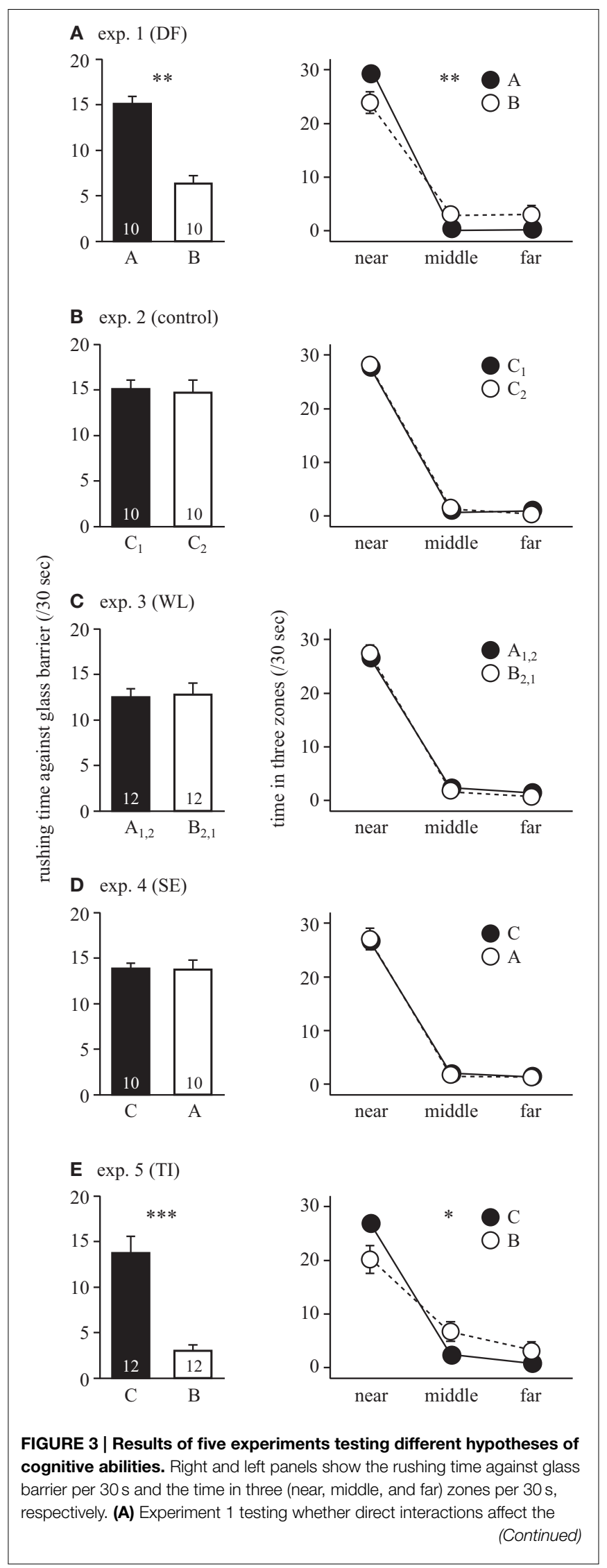




\section{FIGURE 3 | Continued}

next fight between the same individuals. (B) Experiment 2 with two strangers (C) Experiment 3 testing winner/loser effect. (D) Experiment 4 testing eavesdropping effect. (E) Experiment 5 testing transitive inference. See details of fish combinations and expectations from the different hypothesis in Figure 2. Error bars indicate s.e.m. Numerals in bars show sample sizes. Asterisks denote the significant differences in rushing time between fish groups by Wilcoxon signed-rank tests (left panels) and the significant interactions between fish group and zone on the proportion of time stayed in each zone by beta binomial GLZs (right panels): ${ }^{\star} p<0.05 ;{ }^{* \star} p<0.01$; ${ }^{* * *} p<0.001$.

\section{Transitive Inference}

In Experiment 5, we tested whether fish can infer dominance by a combination of observation and their own experience. As expected, fish $\mathrm{B}$, having lost a fight against fish $\mathrm{A}$ after observing fish $\mathrm{C}$ defeating fish $\mathrm{A}$, less frequently attacked fish $\mathrm{C}$ through the glass barrier (Wilcoxon signed-ranks test, $T=0.0, P=0.0002$ ) and spent less time in the near zone than the winning fish $\mathrm{C}$ (beta binomial GLZ, fish group $\times$ zone: $\chi_{2}^{2}=7.53, P=0.02$, Figure 3E). This result confirms that fish $\mathrm{B}$ correctly inferred that it was subordinate to fish $\mathrm{C}$ after watching fish $\mathrm{C}$ defeated fish $\mathrm{A}$, that had defeated fish B. Comparing across experiments, we found that rushing time significantly differed between fish B in Experiment 5 and the loser fish B in Experiment $1(U=23.5$, $P=0.01$ ), but the time in three zones was not different (fish group $\times$ zone: $\chi_{2}^{2}=2.62, P=0.27$, fish group: $\chi_{1}^{2}=0.08, P$ $=0.78$ ). Further, rushing time did not differ between the fish $\mathrm{C}$ in Experiment 5 and the winner fish A in Experiment 1 (MannWhitney $U$-test, $U=46.5, P=0.37$ ), but the time in each zone just differed (beta binomial GLZ, fish group $\times$ zone: $\chi_{2}^{2}=5.98$, $P=0.05)$. Overall in Experiment 5, fish B performed similarly to the losers $\mathrm{B}$ in Experiment 1, and fish $\mathrm{C}$ similarly to the winners $\mathrm{A}$ in Experiment 1 . However, the aggressiveness of fish $\mathrm{C}$ in Experiment 5 was similar to that of stranger $\mathrm{C}_{1}$ and $\mathrm{C}_{2}$ in Experiment 2 that had no information about the dominance relationship ( $z=0.76, P=0.44$ in rushing time, fish group $\times$ zone: $\chi_{2}^{2}=0.51, P=0.78$; fish group: $\chi_{1}^{2}=0.01, P=0.91$ in time in zones).

\section{Discussion}

Our results support the hypothesis that the cichlid Julidochromis transcriptus can infer social status of unknown individuals using transitive inference. Using only observations of a social interaction between a stranger and a known individual, focal animals in our experiments changed their behavior in a manner consistent with having inferred their social status relative to that of a stranger.

Our first experiment showed that fish used direct information from a previous physical encounter to re-establish dominance without additional physical contact, which confirms that our protocol for the test phase is adequate. The second experiment showed that physical encounters are necessary to establish pairwise dominance. Our third experiment clearly showed that winner/loser effects do not operate in this species. In our fourth experiment, the operation of social eavesdropping alone was ruled out, as we observed no differences in the responses of the observing fish against winner of an observed contest (Oliveira et al., 1998; Hsu et al., 2006, 2011). In Experiment 5 , the focal male (B), which had no direct contact with the winning male $(\mathrm{C})$ behaved submissively against $\mathrm{C}$ in test phase, suggesting transitive inference by the focal male. Alternatively, this submissive response might be caused by loser effects or social eavesdropping. However, Experiment 3 demonstrated that loser effects do not cause a submissive response, and Experiment 4 demonstrated that this fish does not use social eavesdropping alone. Any effects of physical characteristics on competition outcomes can also be ruled out because fish were of equal size and sex, and there are no other phenotypic indicators of dominance in this species (e.g., "status badges"; Møller, 1987; Beani and Turillazzi, 1999). Thus, our fifth experiment shows that male J. transcriptus infer the relative social status of an unknown stranger without physical interaction, using transitive inference (Whitehouse, 1997; Hsu et al., 2006, 2011; Oliveira et al., 2009).

\section{Integration of Cognitive Ability from Multiple Sources}

Social eavesdropping and winner/loser effects appear not to operate in J. transcriptus, potentially because transitive inference is a more effective method for determining social relationships. While observation of social interactions is required to obtain social information about strangers during transitive inference, our results suggest that eavesdropping must be accompanied by direct contact with one of the competitors to determine social relationships in J. transcriptus. In highly social animals, individuals repeatedly interact allowing more accurate inference of social dominance of strangers than would be possible using social eavesdropping or winner/loser effects. In contrast, winner/loser effects and social eavesdropping may be effective in less social species, where inferring dominance of strangers using TI would be difficult as the same members rarely encounter each other multiple times (Hsu et al., 2006, 2011). This suggests that in highly social animals, transitive inference, rather than social eavesdropping or winner/loser effects, may be used to infer social structure. We suggest transitive inference may be mutually exclusive from social eavesdropping or winner/loser effects in the majority of species: the former being prevalent in highly social species and the latter in relatively less social species. This prediction is consistent with previous studies of highly social species, e.g., chickens (Hogue et al., 1996), territorial cichlids (Grosenick et al., 2007), river trout (White and Gowan, 2013), and a highly social cichlid M. auratus (Chase et al., 2003), and also with less social species, e.g., the fighting fish B. splendens (Oliveira et al., 1998; McGregor et al., 2001; Witte and Nobel, 2011), paradise fish (Francis, 1983), and green swordtail (Beaugrand and Goulet, 2000). A powerful test of this hypothesis may be achieved using related animal species with different levels of sociality (e.g., Bond et al., 2003; MacLean et al., 2008).

\section{Transitive Inference}

Our results exhibit two noteworthy points in the effects of transitive inference (TI) of this fish: first, the strength of the effect of TI, and second, the ratio of individuals that can effectively use 
TI. The behavior of the losing fish in Experiment 5, which did not have direct experience with its rival, was identical to those of losing fish in Experiment 1, which did have direct experience with its rival. This similarity suggests that fish might infer their relative social dominance using transitive inference as effectively as through direct physical encounters, which will be a novel finding in studies of vertebrate TI. Comparing behavior we also found that the level of aggression toward subordinates by fish that had correctly inferred their social dominance was equal to that of strangers who had no contest experience. Thus, while TI was correctly used to infer that an individual was inferior to its rival, we cannot be certain that individuals correctly inferred that they were dominant to rivals in Experiment 5. This is also the case of other studies on TI in e.g., hens (Hogue et al., 1996) and pinyon jays (Paz-y-Miño et al., 2004).

In our final experiment, almost all individuals (11/12 individuals) used TI and modified their behavior appropriately. This rate of TI use is comparable to other vertebrates, e.g., hens (15/15 individuals, Hogue et al., 1996), great tits (10/10, Peake et al., 2002), and pigeon jays (6/6, Paz-y-Miño et al., 2004), and suggests that $J$. transcriptus uses TI in the complex social groups that exist in nature (Awata et al., 2005). It is worth exploring whether naïve individuals or less socially experienced fish are equally able to use transitive inference. Social experiences are known to influence subsequent behavior in other fish species (Jordan and Brooks, 2012), as well as highly social spiders and many other species (Jordan et al., 2014), and modifying social experiences in future studies will be shed light on the effects of experience and ontogeny on cognitive ability (e.g., Budaev et al., 1999; Frost et al., 2007; Brown and Laland, 2011).

While studies of fish cognitive abilities are rarely compared with those on birds and mammals, recent work suggests that social fish have considerable cognitive ability (e.g., Bshary et al., 2002; Peake and McGregor, 2004; Bshary and Grutter, 2006; Bshary et al., 2006; Hsu et al., 2006, 2011; Bshary, 2011). For example, individual recognition has been documented in many social fishes (e.g., Hert, 1985; Griffiths and Magurran, 1997a,b; Balshine-Earn and Lotem, 1998; Brown and Laland, 2011; Bshary, 2011; Ochi et al., 2012). To predict the social status of strangers using transitive inference, it is necessary to recognize the individuals and recall their social status (Hsu et al., 2006,

\section{References}

Alfieri, M. S., and Dugatkin, L. A. (2011). "Cooperation and cognition in fishes," in Fish cognition and Behaviour, eds C. Brown, K. Laland, and J. Krause (Oxford: Wiley-Blackwell Publ), 258-276.

ASBS/ABS. (2014). Guidelines for the treatment of animals in behavioural research and teaching. Anim. Behav. 99, I-IX. doi: 10.1016/S0003-3472(14)00451-5

Awata, S., and Kohda, M. (2004). Parental roles and the amount of care in a bi-parental substrate brooding cichlid: the effect of size differences within pairs. Behaviour 141, 1135-1149. doi: 10.1163/15685390426 64623

Awata, S., Kohda, M., Shibata, J., Hori, M., and Heg, D. (2010). Group structure, nest size and reproductive success in the cooperatively breeding cichlid Julidochromis ornatus. Ethology 115, 1-13. doi:10.1111/j.1439-0310.2009.01735.x
2011; Grosenick et al., 2007). Although studies of fish memory are even more scarce than those on recognition, there is evidence that fish can remember social information for a considerable time, for example when making mate choice decisions (e.g., Millinski et al., 1990; Dugatkin and Godin, 1993; Griffiths and Magurran, 1997a,b; Dugatkin, 2000; Tebbich et al., 2002), and integrate it with other information in future social contexts (Dugatkin, 2000; Dugatkin and Earley, 2004; Bshary et al., 2006; Frost et al., 2007; Bshary, 2011; Witte and Nobel, 2011; Jordan and Brooks, 2012; Hotta et al., 2015). These studies suggest that advanced cognitive abilities such as transitive inference may occur across fish taxa. A fascinating future research direction will be to compare the use of TI between fish and mammal and bird species (Byrne and Whiten, 1988, 1992; Bond et al., 2003; MacLean et al., 2008) to establish if either group uses TI more accurately to infer ambiguous social relationships, or if the effects of TI on behavior differ. Finally, our study emphasizes the importance of future studies evaluating the operation of social eavesdropping, winner/loser effects and transitive inference at the same time, which can be achieved using the experimental design we employ here.

\section{Author Contributions}

MK and TT developed the study concept. TH, MK, and TT contributed to the study design. Testing, data collection and the data analysis were performed by TH, SA, and LJ. TH, DH, LJ, and MK drafted the manuscript, and TH, LJ, and MK provided critical revisions. All authors approved the final version of manuscript for submission.

\section{Acknowledgments}

We are grateful to Prof. R. Bshary for his advice on the experiment design in this study. This work was financially supported by Grant-in-Aid for Scientific Research (Nos. 25304017 and 23570033, and on Innovative Areas No. 4501) from JSPS in Japan. All experiments were conducted in compliance with the Regulations on Animal Experiments in Osaka City University and the Japan Ethological Society. 
Beani, L., and Turillazzi, S. (1999). Stripes display in hover-wasps (Vespidae: Stenogastrinae): a socially costly status badge. Anim. Behav. 57, 1233-1239. doi: 10.1006/anbe.1999.1101

Beaugrand, J. P., and Goulet, C. (2000). Distinguishing kinds of prior dominance and subordination experiences in males of green swordtail fish (Xiphophorus helerri). Behav. Processes 50, 131-142. doi: 10.1016/S0376-6357(00)00096-6

Benelli, G., Desneux, N., Romano, D., Conte, G., Messing, R. H., and Canale, A. (2015a). Contest experience enhances aggressive behaviour in a fly: when losers learn to win. Sci. Rep. 5:9347. doi: 10.1038/srep09347

Benelli, G., Romano, D., Desneux, N., Messing, R. H., and Canale, A. (2015b). Sex differences in fighting-induced hyperaggression in a fly. Anim. Behav. 104, 165-174. doi: 10.1016/j.anbehav.2015.02.026

Bond, A. B., Kamil, A. C., and Balda, R. P. (2003). Social complexity and transitive inference in corvids. Anim. Behav. 65, 479-487. doi: 10.1006/anbe.2003.2101

Brown, C., and Laland, K. (2011). "Social learning in fishes," in Fish Cognition and Behaviour, eds C. Brown, K. Laland, and J. Krause (Oxford: Wiley-Blackwell Publ), 238-257.

Brown, C., Laland, K., and Krause, J. (2011). Fish Cognition and Behavior, 2nd Edn. Oxford: Wiley-Blackwell Publ.

Bryant, P. E., and Trabasso, T. (1971). Transitive inferences and memory in young children. Nature 232, 456-458. doi: 10.1038/232456a0

Bshary, R. (2011). "Machiavellian intelligence in fish," in Fish Cognition and Behaviour, eds C. Brown, K. Laland, and J. Krause (Oxford: Wiley-Blackwell Publ), 277-297.

Bshary, R., and Grutter, A. S. (2006). Image scoring and cooperation in a cleaner fish mutualism. Nature 441, 975-978. doi: 10.1038/nature04755

Bshary, R., Hohner, A., Ait-el-Djoudi, K., and Fricke, H. (2006). Interspecific communicative and coordinated hunting between groupers and giant moray eels in the Red Sea. PLoS Biol. 4:e431. doi: 10.1371/journal.pbio.0040431

Bshary, R., Wickler, W., and Fricke, H. (2002). Fish cognition: a primate's eye view. Anim. Cogn. 5, 1-13. doi: 10.1007/s10071-001-0116-5

Budaev, S. V., Zworykin, D. D., and Mochek, A. D. (1999). Consistency of individual differences in behaviour of the lion-headed cichlid, Steatocranus casuarius. Behav. Processes 48, 49-55. doi: 10.1016/S0376-6357(99)00068-6

Byrne, R., and Whiten, A. (1988). Machiavellian Intelligence. Oxford: Oxford University Press.

Byrne, R., and Whiten, A. (1992). Cognitive evolution of primates: evidence from tactical deception. Man 27, 609-627. doi: 10.2307/2803931

Chase, I. D., Tovey, C., and Murch, P. (2003). Two's company, three's a crowd: differences in dominance relationships in isolated versus socially embedded pairs of fish. Behaviour 140, 1193-1217. doi: 10.1163/156853903771980558

Congleton, J. L. (2006). Stability of some commonly measured blood-chemistry variables in young person salmonids exposed to a lethal dose of the anaesthetic MS-222. Aquac. Res. 37, 1146-1149. doi: 10.1111/j.1365-2109.2006.01528.x

Dugatkin, L. A. (2000). Bystander effects and the structure of dominance hierarchies. Behav. Ecol. 12, 348-352. doi: 10.1093/beheco/12.3.348

Dugatkin, L. A., and Earley, R. L. (2004). Individual recognition, dominance hierarchies and winner and loser effects. Proc. R. Soc. Lond. B Biol. Sci. 271, 1537-1540. doi: $10.1098 / \mathrm{rspb} .2004 .2777$

Dugatkin, L. A., and Godin, J. J. (1993). Female mate copying in the guppy Poecilia reticulata: age-dependent effects. Behav. Ecol. 4, 289-292. doi: 10.1093/beheco/4.4.289

Engh, A. L., Siebert, E. R., Greenberg, D. A., and Holekamp, K. E. (2005). Patterns of alliance formation and post-conflict aggression indicate spotted hyenas recognize third party relationships. Anim. Behav. 69, 209-217. doi: 10.1016/j.anbehav.2004.04.013

Francis, R. C. (1983). Experimental effects on agonistic behavior in the paradise fish, Macropodus opercularis. Behaviour 85, 292-313. doi: $10.1163 / 156853983$ X00273

Frost, A. J., Winrow-Giffen, A., Ashley, P. L., and Sneddon, L. U. (2007). Plasticity in animal personality traits: does prior experience alter the degree of boldness? Proc. R. Soc. Lond. B Biol. Sci. 274, 333-339. doi: 10.1098/rspb.2006.3751

Gillan, C. A. (1981). Reasoning in the chimpanzee. II. Transitive inference. J. E. Psychol. Anim. Behav. Processes 7, 150-164. doi: 10.1037/0097-7403.7.2.150

Griffiths, S. W., and Magurran, A. E. (1997a). Schooling preferences for familiar fish vary with group size in a wild guppy population. Proc. R. Soc. Lond. B Biol. Sci., 264, 547-551. doi: 10.1098/rspb.1997.0078
Griffiths, S. W., and Magurran, A. E. (1997b). Familiarity in schooling fish: how long does it take to acquire? Anim. Behav. 53, 945-949. doi: 10.1006/anbe.1996.0315

Grosenick, L., Clement, T. S., and Fernald, R. D. (2007). Fish can infer social rank by observation alone. Nature 445, 429-432. doi: 10.1038/nature05511

Heg, D., and Bachar, Z. (2006). Cooperative breeding in the Lake Tanganyika cichlid Julidochromis ornatus. Environ. Biol. Fish 76, 265-281. doi: 10.1007/s10641-006-9032-5

Hert, E. (1985). Individual recognition of helpers by the breeders in the cichlid fish Lamprologus brichardi (Poll, 1974). Z. Tierpsychol. 68, 313-325. doi: 10.1111/j.1439-0310.1985.tb00132.x

Hogue, M. E., Beaugrand, J. P., and Laguë, P. C. (1996). Coherent use of information by hens observing their former dominant defeating or being defeated by a stranger. Behav. Processes 38, 241-252. doi: 10.1016/S03766357(96)00035-6

Hotta, T., Jordan, L. A., Takeyama, T., and Kohda, M. (2015). Order effects in transitive inference: does the presentation order of social information affect transitive inference in social animals. Front. Ecol. Evol. 3:59. doi: $10.3389 /$ fevo.2015.00059

Hotta, T., Takeyama, T., Jordan, L. A., and Kohda, M. (2014). Duration of memory of dominance relationships in a group living cichlid. Naturwissenschaften 101, 745-751. doi: 10.1007/s00114-014-1213-z

Hsu, Y., Earley, R. L., and Wolf, L. L. (2006). Modulation of aggressive behaviour by fighting experience: mechanism and contest outcomes. Biol. Rev. 81, 33-74. doi: $10.1017 /$ S146479310500686X

Hsu, Y., Earley, R. L., and Wolf, L. L. (2011). "Aggressive behaviour in fish: integrating information about contest costs," in Fish Cognition and Behaviour, eds C. Brown, K. Laland, and J. Krause (Oxford: Wiley-Blackwell Publ), 108-134.

Huntingfold, F., and Turner, A. (1987). Animal Conflict. London: Chapman and Hall.

Jordan, L. A., Avolio, C., Herbert-Read, J. E., Krause, J., Rubenstein, D., and Ward, A. (2010a). Group structure in a restricted entry system is mediated by both resident and joiner preferences. Behav. Ecol. Sociobiol. 64, 1099-1106. doi: 10.1007/s00265-010-0924-1

Jordan, L. A., and Brooks, R. C. (2012). Recent social history alters male courtship preferences. Evolution 66, 280-287. doi: 10.1111/j.1558-5646.2011.01421.x

Jordan, L. A., Kokko, H., and Kasumovic, M. M. (2014). Reproductive foragers: spider males choose mates by selecting among available competitive environments. Am. Natural. 183, 638-649. doi: 10.1086/675755

Jordan, L. A., Wong, M. Y. L., and Balshine, S. (2010b). The effects of familiarity and social hierarchy on group membership decisions in a social fish. Biol. Lett. 6, 301-303. doi: 10.1098/rsbl.2009.0732

Kohda, M., Heg, D., Makino, Y., Takeyama, T., Shibata, J., Watanabe, K., et al. (2009). Living on the wedge: female control of paternity in a cooperatively polyandrous cichlid. Proc. R. Soc. Lond. B Biol. Sci. 276, 4207-4214. doi: 10.1098/rspb.2009.1175

MacLean, E. L., Merritt, D. J., and Brannon, E. M. (2008). Social complexity predicts transitive reasoning in prosimian primates. Anim. Behav. 76, 479-486. doi: 10.1016/j.anbehav.2008.01.025

McGregor, P. K., Peake, T. M., and Lampe, H. M. (2001). Fighting fish Betta splendens extract relative information from apparent interactions: what happens when what you see is not what you get. Anim. Behav. 62, 1059-1065. doi: 10.1006/anbe.2001.1850

Millinski, M., Kulling, D., and Kettler, R. (1990). Tit for tat: sticklebacks Gasterostues aculeatus 'trusting' a cooperative partner. Behav. Ecol. 1, 7-11. doi: 10.1093/beheco/1.1.7

Møller, A. P. (1987). Variation in badge size in male house sparrows Passer domesticus: evidence for status signaling. Anim. Behav. 35, 1637-1644. doi: 10.1016/S0003-3472(87)80056-8

Ochi, H., Awata, S., and Kohda, M. (2012). Differential attack by a cichlid fish on resident and non-resident fish of another cichlid species. Behaviour 149, 99-109. doi: 10.1163/156853912X6 29139

Oliveira, R. F., McGregor, P. K., and Latruffe, C. (1998). Know thine enemy: fighting fish gather information from observing conspecific interactions. Proc. R. Soc. Lond. B Biol. Sci. 265, 1045-1049. doi: 10.1098/rspb.1998.0397 
Oliveira, R. F., Silva, A., and Canário, A. V. M. (2009). Why do winners keep winning? Androgen mediation of winner but not loser effects in cichlid fish. Proc. R. Soc. Lond. B Biol. Sci. 276, 2249-2256. doi: 10.1098/rspb.2009.0132

Paz-y-Miño, C. G., Bond, A. B., Kamil, A., and Balda, R. P. (2004). Pinyon jays use transitive inference to predict social dominance. Nature 430, 778-781. doi:10.1038/nature02723

Peake, T. M., and McGregor, P. K. (2004). Information and aggression in fishes. Anim. Learn. Behav. 32, 114-121. doi: 10.3758/BF03196012

Peake, T. M., Terry, A. M. R., McGregor, P. K., and Dabelsteen, T. (2002). Do great tits assess rivals by combining direct experience with information gathered by eavesdropping? Proc. R. Soc. Lond. B Biol. Sci. 269, 1925-1929. doi: 10.1098/rspb.2002.2112

Sopinka, N. M., Fitzpatrick, J. L., Desjardins, J. K., Stiver, K. A., Marsh-Rollo, S. E., and Balshine, S. (2009). Liver size reveals social status in the African cichlid Neolamprologus pulcher. J. Fish Biol. 75, 1-16. doi: 10.1111/j.10958649.2009.02234.x

Tebbich, S., Bshary, R., and Grutter, A. S. (2002). Cleaner fish Labroides dimidiatus recognize familiar clients. Anim. Cogn. 5, 139-145. doi: 10.1007/s10071-0020141-z

Vasconcelos, M. (2008). Transitive inference in non-human animals: an empirical and theoretical analysis. Behav. Processes 78, 313-334. doi: 10.1016/j.beproc.2008.02.017

Wallen, K., and Wojciechowski-Metzlar, C. I. (1985). Social conditioning and dominance in male Betta splendens. Behav. Processes 11, 181-188. doi: 10.1016/0376-6357(85)90059-2
White, S. L., and Gowan, C. (2013). Brook trout use individual recognition and transitive inference to determine social rank. Behav. Ecol. 24, 63-69. doi: 10.1093/beheco/ars136

Whitehouse, M. E. A. (1997). Experience influences male-male contests in the spider Argyrodes antipodiana (Theridiidae: Araneaea). Anim. Behav. 53, 913-923. doi: 10.1006/anbe.199 6.0313

Witte, K., and Nobel, S. (2011). "Learning and mate choice," in Fish Cognition and Behaviour, eds C. Brown, K. Laland, and J. Krause (Oxford: Wiley-Blackwell Publ), 81-107.

Wong, M., and Balshine, S. (2010). The evolution of cooperative breeding in the African cichlid fish, Neolamprologus pulcher. Biol. Rev. 86, 511-530. doi: 10.1111/j.1469-185X.2010.00158.x

Conflict of Interest Statement: The authors declare that the research was conducted in the absence of any commercial or financial relationships that could be construed as a potential conflict of interest.

Copyright (c) 2015 Hotta, Takeyama, Heg, Awata, Jordan and Kohda. This is an open-access article distributed under the terms of the Creative Commons Attribution License (CC BY). The use, distribution or reproduction in other forums is permitted, provided the original author(s) or licensor are credited and that the original publication in this journal is cited, in accordance with accepted academic practice. No use, distribution or reproduction is permitted which does not comply with these terms. 